\title{
A Note on $\operatorname{Card}(X)$
}

Weituo Dai, Meng Wang* and Limin Sun

School of Mathematical Sciences, Zhejiang University, Hangzhou, Zhejiang 310027, China

Received 17 September 2020; Accepted (in revised version) 21 September 2020

Dedicated to Professor Weiyi Su on the occasion of her 80th birthday

Abstract. The main interests here are to study the relationship between $\operatorname{card}(X)$ and $\operatorname{card}(\mathcal{P}(X))$ and the connection between the separability of a space $X$ and cardinality of some function space on it. We will convert the calculation of $\operatorname{card}(\mathcal{P}(X))$ to the calculation of $\operatorname{card}(\mathcal{F}(X \rightarrow \mathbb{Q}))$. The main tool we used here is Zorn Lemma.

Key Words: Cardinality, separability of space, Zorn Lemma.

AMS Subject Classifications: 03E10

\section{Introduction}

Let $X$ be a set. If $X$ is a finite set, we call the number of elements of $X$ the cardinality of $X$, and denote it by $\operatorname{card}(X)$. For two infinite sets $X$ and $Y$, we can use this notion to compare the "number" of two sets $X$ and $Y$. The following expressions are well-known:

(i) $\operatorname{card}(X) \leq \operatorname{card}(Y)$ if there exists an injective map $\phi: X \rightarrow Y$;

(ii) $\operatorname{card}(X) \geq \operatorname{card}(Y)$ if there exists a surjective map $\phi: X \rightarrow Y$;

(iii) $\operatorname{card}(X)=\operatorname{card}(Y)$ if there exists a bijective map $\phi: X \rightarrow Y$.

Let $X$ and $Y$ be two sets. We recall the following theorems in [1-3].

Theorem 1.1. $\operatorname{card}(X)=\operatorname{card}(Y)$ if and only if $\operatorname{card}(X) \leq \operatorname{card}(Y)$ and $\operatorname{card}(X) \geq \operatorname{card}(Y)$ both hold.

Theorem 1.2. Either $\operatorname{card}(X)<\operatorname{card}(Y) \operatorname{or} \operatorname{card}(Y)<\operatorname{card}(X)$ or $\operatorname{card}(X)=\operatorname{card}(Y)$.

Theorem 1.3. $\operatorname{card}(X)<\operatorname{card}(\mathcal{P}(X))$.

*Corresponding author. Email address: mathdreamcn@zju.edu.cn (M. Wang) 
In this paper, we use $\mathbb{N}, \mathbb{Z}, \mathbb{Q}, \mathbb{R}$ and $\mathbb{C}$ to denote the set of positive integers, integers, rational numbers, real numbers and complex numbers respectively. The number filed $F$ mentioned here is a subfield of $\mathbb{C}$, thus $\mathbb{Q}$ is the minimal number field and $F \supset \mathbb{Q}$. Given two sets $X$ and $Y$, we denote

$$
\mathcal{F}(X \rightarrow Y)=\{\operatorname{map} f: X \rightarrow Y\} .
$$

Especially, there is a natural algebra structure on $\mathcal{F}(X \rightarrow F)$ if $F$ is a field. As usual, we use $(X, \rho)$ to denote a metric space with a metric map $\rho: X \times X \rightarrow[0,+\infty)$, which satisfies

(i) $\rho\left(x_{1}, x_{2}\right)=0$ if and only if $x_{1}=x_{2}$;

(ii) $\rho\left(x_{1}, x_{2}\right)=\rho\left(x_{2}, x_{1}\right)$;

(iii) $\rho\left(x_{1}, x_{3}\right) \leq \rho\left(x_{1}, x_{2}\right)+\rho\left(x_{2}, x_{3}\right)$, where $x_{1}, x_{2}, x_{3}$ are arbitrary points of $X$.

We use $(X, \mathcal{M}, \mu)$ to denote a measure space, where $\mathcal{M}$ is a $\sigma$-algebra on $X$, and $\mu$ is a measure, i.e., $\mu: \mathcal{M} \rightarrow[0,+\infty]$ is a map, satisfying

(i) $\mu(\phi)=0$;

(ii) $\mu\left(\cup_{j=1}^{\infty} E_{j}\right)=\sum_{j=1}^{+\infty} \mu\left(E_{j}\right)$, where $E_{j} \in \mathcal{M}$ and $E_{j_{1}} \cap E_{j_{2}}=\varnothing$, $\left(j_{1} \neq j_{2}\right)$.

We denote $\operatorname{card}(\mathbb{N})=c_{0}$, which is the minimal cardinality of all infinite sets. Denote $\operatorname{card}(\mathbb{R})=c$, which is called "cardinality of the continuum".

Let $X$ and $Y$ be two sets and $\alpha=\operatorname{card}(X), \beta=\operatorname{card}(Y)$. We have the following definitions,

Definition 1.1. If $X \cap Y=\varnothing$, we define $\alpha+\beta=\operatorname{card}(X \cup Y)$.

Definition 1.2. Define $\alpha \cdot \beta=\operatorname{card}(X \times Y)$.

Definition 1.3. Define $\beta^{\alpha}=\operatorname{card}(\mathcal{F}(X \rightarrow Y))$.

We verify that these three definitions are well-defined. Suppose two sets $X_{1}$ and $Y_{1}$ satisfy $\operatorname{card}\left(X_{1}\right)=\operatorname{card}(X), \operatorname{card}\left(Y_{1}\right)=\operatorname{card}(Y)$ and $X_{1} \cap Y_{1}=\varnothing$ (in Definition 1.1). Then, we have bijective maps $\phi: X \rightarrow X_{1}$ and $\psi: Y \rightarrow Y_{1}$. We construct three maps $\omega, \theta$, $\eta$ as follows:

$$
\begin{aligned}
& \omega: X \cup Y \rightarrow X_{1} \cup Y_{1}, \quad \omega(z)= \begin{cases}\phi(x), & \text { if } z=x \in X, \\
\psi(y), & \text { if } z=y \in Y,\end{cases} \\
& \theta: X \times Y \rightarrow X_{1} \times Y_{1}: \theta(x, y)=(\phi(x), \psi(y)),
\end{aligned}
$$

where $x \in X, y \in Y$.

$$
\eta: \mathcal{F}(X \rightarrow Y) \rightarrow \mathcal{F}\left(X_{1} \rightarrow Y_{1}\right): \eta(f)=\psi \circ f \circ \phi^{-1},
$$

where $f \in \mathcal{F}(X \rightarrow Y)$, "o" represents the composition of maps. It is easy to verify that $\omega, \theta, \eta$ are bijective. Thus these definitions are well-defined. 
Remark 1.1. (1) Note that in Definition 1.1, if $\beta=\alpha$, we have $\alpha+\alpha=\operatorname{card}(X \times\{0,1\})$, where $\alpha=\operatorname{card}(X)$. (2) In some literature, $2^{\alpha}=\operatorname{card}(\mathcal{P}(X))$ where $\alpha=\operatorname{card}(X)$. We will see that this coincides with the Definition 1.3, which will be explained in the Theorem 1.8 .

We state the theorems about the cardinal computation as follows and leave the discussion about cardinality and the separability of a space $X$ in Section 3.

Theorem 1.4. If $\alpha_{1}, \alpha_{2}, \beta$ are cardinal numbers of three nonempty sets, then $\beta^{\alpha_{1}} \cdot \beta^{\alpha_{2}}=\beta^{\alpha_{1}+\alpha_{2}}$.

Theorem 1.5. If $\alpha, \beta_{1}, \beta_{2}$ are cardinal numbers of three nonempty sets, then $\beta_{1}^{\alpha} \cdot \beta_{2}^{\alpha}=\left(\beta_{1} \cdot \beta_{2}\right)^{\alpha}$.

Theorem 1.6. Given two cardinal numbers $\alpha, \beta$, if at least one of them is the cardinal number of an infinite set, then $\alpha+\beta=\max (\alpha, \beta)$. Especially, $\alpha+\alpha=\alpha$ when $\alpha$ is the cardinal number of an infinite set.

Theorem 1.7. If $\alpha, \beta$ are cardinal numbers of two nonempty sets and at least one of them is infinite, then $\alpha \cdot \beta=\max (\alpha, \beta)$. Especially, $\alpha \cdot \alpha=\alpha$ when $\alpha$ is the cardinal number of an infinite set.

Theorem 1.8. Suppose two cardinal numbers $\alpha, \beta$ satisfy $\alpha \geq \beta \geq 2$, and $\alpha \geq c_{0}$. Then

$$
\beta^{\alpha}=2^{\alpha}=\operatorname{card}(\mathcal{P}(X)),
$$

where $\alpha=\operatorname{card}(X)$.

Theorem 1.9. Suppose $V$ is an infinite-dimensional linear space over the field $F$ with a basis $E$. Then, $\operatorname{card}(V)=\max (\operatorname{card}(E), \operatorname{card}(F))$. Especially, $\operatorname{card}(V)=\operatorname{card}(E)$ when $F=\mathbb{Q}$.

Example 1.1. Let $V=\mathbb{R}, F=\mathbb{Q}$. We know that

$$
e=\sum_{n=0}^{\infty} \frac{1}{n !}
$$

is a transcendental number, so $\left\{e^{k}\right\}_{k=0}^{\infty}$ is a linear independent set over $\mathbb{Q}$, thus $\mathbb{R}$ is an infinite-dimensional linear space over $\mathbb{Q}$. If $E$ is a basis of $\mathbb{R}$ over $\mathbb{Q}$, then $\operatorname{card}(E)=$ $\operatorname{card}(\mathbb{R})=c$.

Theorem 1.10. For any two infinite sets $X$ and $Y, \operatorname{card}(X)=\operatorname{card}(Y)$ if and only if $\operatorname{card}(\mathcal{P}(X))=$ $\operatorname{card}(\mathcal{P}(Y))$ and there exists an algebra isomorphism $\Psi: \mathcal{F}(X \rightarrow \mathbb{Q}) \rightarrow \mathcal{F}(Y \rightarrow \mathbb{Q})$.

This paper is organized as follows. In Section 2, we prove Theorems 1.4-1.10. In Section 3, we discuss the connection between separability of a space $X$ and the cardinality of some function space on it and prove several related results. Finally, several unsolved questions are raised in the context. 


\section{Proof of Theorems 1.4-1.10}

Proof of Theorem 1.4. Suppose $X_{1}, X_{2}, Y$ are nonempty sets satisfying $\operatorname{card}\left(X_{1}\right)=\alpha_{1}$, $\operatorname{card}\left(X_{2}\right)=\alpha_{2}$ and $\operatorname{card}(Y)=\beta$, without loss of generality, we can assume that $X_{1} \cap X_{2}=$ $\varnothing$. According to Definition 1.3,

$$
\begin{aligned}
& \beta^{\alpha_{1}}=\operatorname{card}\left(\mathcal{F}\left(X_{1} \rightarrow Y\right)\right), \quad \beta^{\alpha_{2}}=\operatorname{card}\left(\mathcal{F}\left(X_{2} \rightarrow Y\right)\right), \\
& \begin{aligned}
\beta^{\alpha_{1}} \cdot \beta^{\alpha_{2}} & =\operatorname{card}\left(\mathcal{F}\left(X_{1} \rightarrow Y\right) \times \mathcal{F}\left(X_{2} \rightarrow Y\right)\right) \\
& =\left\{\left(f_{1}, f_{2}\right): \operatorname{map} f_{1}: X_{1} \rightarrow Y, \operatorname{map} f_{2}: X_{2} \rightarrow Y\right\} .
\end{aligned}
\end{aligned}
$$

Since $X_{1} \cap X_{2}=\varnothing,\left(f_{1}\left(x_{1}\right), f_{2}\left(x_{2}\right)\right), x_{1} \in X_{1}, x_{2} \in X_{2}$, corresponds bijectively to map $f: X_{1} \cup X_{2} \rightarrow Y$, where

$$
f(x)= \begin{cases}f_{1}\left(x_{1}\right), & \text { if } x=x_{1} \in X_{1} \\ f_{2}\left(x_{2}\right), & \text { if } x=x_{2} \in X_{2}\end{cases}
$$

Therefore, $\mathcal{F}\left(X_{1} \rightarrow Y\right) \times \mathcal{F}\left(X_{2} \rightarrow Y\right)=\mathcal{F}\left(X_{1} \cup X_{2} \rightarrow Y\right)$. By Definition 1.1 and Definition 1.3 , we have

$$
\beta^{\alpha_{1}} \cdot \beta^{\alpha_{2}}=\operatorname{card}\left(\mathcal{F}\left(X_{1} \cup X_{2} \rightarrow Y\right)\right)=\beta^{\operatorname{card}\left(X_{1} \cup X_{2}\right)}=\beta^{\alpha_{1}+\alpha_{2}} .
$$

Thus, we complete the proof.

Proof of Theorem 1.5. Suppose $X, Y_{1}, Y_{2}$ are nonempty sets satisfying $\operatorname{card}(X)=\alpha$, $\operatorname{card}\left(Y_{1}\right)=\beta_{1}$ and $\operatorname{card}\left(Y_{2}\right)=\beta_{2}$. By Definition 1.3, $\beta_{1}^{\alpha}=\operatorname{card}\left(\mathcal{F}\left(X \rightarrow Y_{1}\right)\right), \beta_{2}^{\alpha}=$ $\operatorname{card}\left(\mathcal{F}\left(X \rightarrow Y_{2}\right)\right)$. By Definition 1.2, $\beta_{1}^{\alpha} \cdot \beta_{2}^{\alpha}=\operatorname{card}\left(\mathcal{F}\left(X \rightarrow Y_{1}\right) \times \mathcal{F}\left(X \rightarrow Y_{2}\right)\right)$. Besides $\mathcal{F}\left(X \rightarrow Y_{1}\right) \times \mathcal{F}\left(X \rightarrow Y_{2}\right)=\left\{\operatorname{map} f: X \rightarrow Y_{1} \times Y_{2}\right\}=\mathcal{F}\left(X \rightarrow Y_{1} \times Y_{2}\right)$. By Definition $1.2, \operatorname{card}\left(Y_{1} \times Y_{2}\right)=\beta_{1} \cdot \beta_{2}$. It follows that

$$
\beta_{1}^{\alpha} \beta_{2}^{\alpha}=\operatorname{card}\left(\mathcal{F}\left(X \rightarrow Y_{1} \times Y_{2}\right)\right)=\left(\operatorname{card}\left(Y_{1} \times Y_{2}\right)\right)^{\operatorname{card}(X)}=\left(\beta_{1} \cdot \beta_{2}\right)^{\alpha},
$$

the second "=" follows by Definition 1.2.

Proof of Theorem 1.6. We first consider the case when $\alpha+\alpha=\alpha$, where $\alpha$ is the cardinal number of an infinite set. The proof of this case can be seen in [3, Page 30].

To prove the general result, we divide the situation into two cases:

(i) $\beta>\alpha$ and $\alpha$ is the cardinal number of an infinite set. Suppose $X, Y$ satisfy $X \cap Y=\varnothing$, $\operatorname{card}(X)=\alpha$ and $\operatorname{card}(Y)=\beta$. Since $\beta>\alpha$, there is a proper subset $Y_{1}$ of $Y$ s.t. $\operatorname{card}\left(Y_{1}\right)=$ $\operatorname{card}(X)=\alpha$ and $Y_{1} \cap X=\varnothing$. Because $\alpha+\alpha=\alpha$, we obtain $\operatorname{card}\left(X \cup Y_{1}\right)=\operatorname{card}\left(Y_{1}\right)=\alpha$. So there is a bijective map $\phi: X \cup Y_{1} \rightarrow Y_{1}$. We construct a map $\psi: X \cup Y=\left(X \cup Y_{1}\right) \cup$ $\left(Y \backslash Y_{1}\right) \rightarrow Y:$

$$
\psi(z)= \begin{cases}\phi(z), & \text { if } z \in X \cup Y_{1} \\ z, & \text { if } z \in Y \backslash Y_{1} .\end{cases}
$$


Then $\psi$ is trivially bijective, thus $\operatorname{card}(X \cup Y)=\operatorname{card}(Y)$, which indicates $\alpha+\beta=\beta=$ $\max (\alpha, \beta)$.

(ii) $\beta>\alpha$, where $\alpha$ is the cardinal number of a finite set, $\beta$ is the cardinal number of an infinite set. Suppose $X=\left\{x_{1}, \cdots, x_{k}\right\}, Y$ is an infinite set, $\beta=\operatorname{card}(Y)$ and $X \cap Y=\varnothing$. Select a sequence $\left\{y_{j}\right\}_{j=1}^{\infty}$ in $Y$. Thus,

$$
X \cup Y=\left(\left\{y_{j}\right\}_{j=1}^{\infty} \cup\left\{x_{1}, \cdots, x_{k}\right\}\right) \cup\left(Y \backslash\left\{y_{j}\right\}_{j=1}^{\infty}\right) .
$$

As any infinite countable set has card $c_{0}$, there exists bijection $\eta:\left\{y_{j}\right\}_{j=1}^{\infty} \cup\left\{x_{1}, \cdots, x_{k}\right\} \rightarrow$ $\left\{y_{j}\right\}_{j=1}^{\infty}$. Let $\omega: X \cup Y \rightarrow Y$ be defined as

$$
\omega(z)= \begin{cases}\eta(z), & \text { if } z \in\left\{y_{j}\right\}_{j=1}^{\infty} \cup\left\{x_{1}, \cdots, x_{k}\right\} \\ z, & \text { if } z \in Y \backslash\left\{y_{j}\right\}_{j=1}^{\infty} .\end{cases}
$$

It is easy to see that $\omega$ is bijective, so $\operatorname{card}(X \cup Y)=\operatorname{card}(Y)$. We conclude that $\alpha+\beta=$ $\beta=\max (\alpha, \beta)$.

Remark 2.1. Applying the principal of induction, the special case in Theorem $1.6 \alpha+\alpha=$ $\alpha$ can be extended to

$$
\underbrace{\alpha+\cdots+\alpha}_{k}=\alpha
$$

where $k \in \mathbb{N}, \alpha$ is the cardinal number of an infinite set.

Proof of Theorem 1.7. We first consider the case when $\alpha \cdot \alpha=\alpha$, where $\alpha$ is the cardinal of an infinite set. Suppose $X$ satisfies $\operatorname{card}(X)=\alpha$. Define

$$
X=\{(A, \phi): A \subset X \text { satisfies } \operatorname{card}(A \times A)=\operatorname{card}(A), \phi: A \rightarrow A \times A \text { bijective }\} .
$$

Since $X$ is infinite, it has countably infinite subset $A_{0}=\left\{x_{j}\right\}_{j=1}^{\infty} \subset X$ and we have bijection $\phi_{0}: A_{0} \rightarrow A_{0} \times A_{0}$. Thus, $X$ is nonempty. We can define the order relation in $X$ : $\left(A_{1}, \phi_{1}\right)<\left(A_{2}, \phi_{2}\right) \Leftrightarrow A_{1} \subset A_{2}$ and $\left.\phi_{2}\right|_{A_{1}}=\phi_{1}$. Suppose $X_{1}$ is a totally ordered subset of $X$. Let $\tilde{A}=\cup_{A \in X_{1}} A$, then

$$
\tilde{A} \times \tilde{A}=\cup_{A \in X_{1}, B \in X_{1}}(A \times B)=\cup_{A \in X_{1}}(A \times A) .
$$

Construct the map $\tilde{\phi}: \tilde{A} \rightarrow \tilde{A} \times \tilde{A}$, where $\tilde{\phi}(x)=\phi(x)$, if $x \in A \in X_{1}$. So $(\tilde{A}, \tilde{\phi}) \in X$ and $(\tilde{A}, \tilde{\phi})$ is an upper bound for $X_{1}$ in $X$. By Zorn Lemma, $X$ has maximal element $\left(A^{*}, \phi^{*}\right)$. Here are three cases as follows:

(i) $X \backslash A^{*}=\varnothing$, i.e., $X=A^{*}$. Then $\alpha \cdot \alpha=\alpha$. 
(ii) $\operatorname{card}\left(X \backslash A^{*}\right) \leq \operatorname{card}\left(A^{*}\right)$. Let $B=X \backslash A^{*}$, then $\operatorname{card}(B) \leq \operatorname{card}\left(A^{*}\right), X=A^{*} \cup B$ and $A^{*} \cap B=\varnothing$. By Theorem 1.6,

$$
\alpha=\operatorname{card}(X)=\operatorname{card}\left(A^{*} \cup B\right)=\max \left(\operatorname{card}\left(A^{*}\right), \operatorname{card}(B)\right)=\operatorname{card}\left(A^{*}\right) .
$$

So $\left(A^{*}, \phi^{*}\right) \in X, \operatorname{card}\left(A^{*}\right)=\operatorname{card}\left(A^{*} \times A^{*}\right)$. It follows that $\alpha \cdot \alpha=\alpha$.

(iii) $\operatorname{card}\left(X \backslash A^{*}\right)>\operatorname{card}\left(A^{*}\right)$. This case cannot occur because of the reason that: If $\operatorname{card}\left(X \backslash A^{*}\right)>\operatorname{card}\left(A^{*}\right)$, then $X$ has proper subset $B \subset X \backslash A^{*}$ such that $\operatorname{card}(B)=$ $\operatorname{card}\left(A^{*}\right)$, i.e., there exists a bijection from $B$ to $A^{*}$, so $\operatorname{card}\left(A^{*} \times B\right)=\operatorname{card}(B \times$ $\left.A^{*}\right)=\operatorname{card}(B \times B)=\operatorname{card}\left(A^{*} \times A^{*}\right)$. Since $B \cap A^{*}=\varnothing$, any two of the four product sets in the above equation do not intersect. Note the following equality:

$$
\left(A^{*} \cup B\right) \times\left(A^{*} \cup B\right)=\left(A^{*} \times A^{*}\right) \cup\left\{\left(A^{*} \times B\right) \cup\left(B \times A^{*}\right) \cup(B \times B)\right\} .
$$

Applying Theorem 1.6 and the result in Remark 2.1, we obtain following equality:

$$
\begin{aligned}
& \operatorname{card}\left(\left\{\left(A^{*} \times B\right) \cup\left(B \times A^{*}\right) \cup(B \times B)\right\}\right) \\
= & \operatorname{card}\left(A^{*} \times A^{*}\right)=\operatorname{card}\left(A^{*}\right)=\operatorname{card}(B) .
\end{aligned}
$$

Thus, there is a bijection $\eta: B \rightarrow\left\{\left(A^{*} \times B\right) \cup\left(B \times A^{*}\right) \cup(B \times B)\right\}$. We construct the map $\omega: A^{*} \cup B \rightarrow\left(A^{*} \cup B\right) \times\left(A^{*} \cup B\right)$ as follows:

$$
\omega(z)= \begin{cases}\phi^{*}(z), & \text { if } z \in A^{*}, \\ \eta(z), & \text { if } z \in B\end{cases}
$$

Then $\omega$ is bijective and $\left.\omega\right|_{A^{*}}=\phi^{*}$. Therefore, $\left(A^{*} \cup B, \omega\right) \in X$ and $\left(A^{*} \cup B, \omega\right)>$ $\left(A^{*}, \phi^{*}\right)$. This contradicts that $\left(A^{*}, \phi^{*}\right)$ is a maximal element for $X$, it follows that $\alpha \cdot \alpha=\alpha$.

Now we turn to the general case. Suppose $\beta>\alpha, X, Y$ satisfy $\operatorname{card}(X)=\alpha, \operatorname{card}(Y)=\beta$, where $\beta$ is the cardinal number of an infinite set. Without loss of generality, assume $X \cap Y=\varnothing$. Since $X$ is nonempty, $\exists x_{1} \in X$, so $\alpha \cdot \beta=\operatorname{card}(X \times Y) \geq \operatorname{card}\left(\left\{x_{1}\right\} \times Y\right)=$ $\operatorname{card}(Y)=\beta$. On the other hand, because $\beta>\alpha, \exists$ proper subset $Y_{1}$ of $Y$ and a bijection $\psi: X \rightarrow Y_{1}$. Let $X_{1}=X \cup\left(Y \backslash Y_{1}\right)$, then $X_{1} \supset X$ and we can construct a bijection $\theta: X_{1} \rightarrow Y$, where

$$
\theta(z)= \begin{cases}\psi(x), & \text { if } z=x \in X \\ z, & \text { if } z \in Y \backslash Y_{1}\end{cases}
$$

Since $\beta \cdot \beta=\beta, \alpha \cdot \beta=\operatorname{card}(X \times Y) \leq \operatorname{card}\left(X_{1} \times Y\right)=\beta \cdot \beta=\beta$. Thus, $\alpha \cdot \beta=\beta=$ $\max (\alpha, \beta)$.

Remark 2.2. Suppose $\alpha$ is the cardinal number of an infinite set. Applying the principal of induction, the special case in Theorem $1.7 \alpha \cdot \alpha=\alpha$ can be extended to $\alpha^{k}=\alpha \cdot \alpha \cdots \alpha=\alpha$, $k \in \mathbb{N}$. Applying Theorem 1.7, the result in Remark 2.1 can be extended to $c_{0} \alpha=\alpha+\alpha+$ $\cdots+\alpha+\cdots=\max \left(c_{0}, \alpha\right)=\alpha$. 
Question 2.1. Whether $\alpha^{k}=\alpha$ can be extended to $\alpha^{c_{0}}=\alpha$ for any $\alpha>c_{0}$ ? If not, for which kind of $\alpha, \alpha^{c_{0}}=\alpha$ holds?

When $\alpha=c_{0}, c_{0}{ }^{c_{0}}=c>c_{0}$; if $\alpha=c$, then $c^{c_{0}}=c$, the proof of which can be found in Theorem 3.2 in the Section 3. For a more common case, suppose $\gamma_{0}=c, \gamma_{k}=2^{\gamma_{k-1}}$, $k \in \mathbb{N}$. If $\alpha \in\left\{\gamma_{0}, \gamma_{1}, \cdots, \gamma_{n}, \cdots\right\}$, then $\alpha^{c_{0}}=\alpha$ holds. The more general case remains to be answered.

Before giving the proof of Theorem 1.8, we give some explanations. Theorem 1.8 means that given an infinite set $X$, then for any set $Y$ satisfying $\operatorname{card}(X) \geq \operatorname{card}(Y) \geq 2$, we have $\operatorname{card}(\mathcal{P}(X))=\operatorname{card}(\mathcal{F}(X \rightarrow Y))$. Especially, let $Y=\{0,1\}$, then $\mathcal{F}(X \rightarrow\{0,1\})=$ $\left\{\right.$ characteristic map $\left.\chi_{E}: E \in \mathcal{P}(X)\right\}$. Therefore, $\operatorname{card}(\mathcal{P}(X))=\operatorname{card}(\mathcal{F}(X \rightarrow Y))$, when computing the cardinal number of $\mathcal{P}(X)$, the choice of $Y$ is flexible to a certain extend.

Proof of Theorem 1.8. Since $\operatorname{card}(Y) \geq 2$, without loss of generality, assume $\{0,1\} \subset Y$. Then $\operatorname{card}(\mathcal{F}(X \rightarrow Y)) \geq \operatorname{card}\left(\left\{\chi_{E}: E \in \mathcal{P}(X)\right\}\right)=\operatorname{card}(\mathcal{P}(X))$. On the other hand, each $f \in \mathcal{F}(X \rightarrow Y)$ has a graph $\{(x, f(x)): x \in X\} \in \mathcal{P}(X \times Y)$. Thus $\operatorname{card}(\mathcal{F}(X \rightarrow Y)) \leq$ $\operatorname{card}(\mathcal{P}(X \times Y))=2^{\operatorname{card}(X \times Y)} ;$ according to Theorem 1.7 and $\operatorname{card}(X) \geq \operatorname{card}(Y) \geq 2$, $\operatorname{card}(X \times Y)=\operatorname{card}(X)$; so $\operatorname{card}(\mathcal{F}(X \rightarrow Y)) \leq 2^{\operatorname{card}(X)}=\operatorname{card}(\mathcal{P}(X))$. In a word, by Theorem 1.1 we get $\operatorname{card}(\mathcal{F}(X \rightarrow Y))=\operatorname{card}(\mathcal{P}(X))$.

Proof of Theorem 1.9. Suppose $V$ is an infinite-dimensional linear space over the field $F$ with a basis $E$. Any element $x \in V$ can be written as $x=\sum_{j=1}^{n} \lambda_{j} e_{j}$ in a unique way, where $\lambda_{j} \in F, e_{j} \in E, e_{j_{1}} \neq e_{j_{2}},\left(j_{1} \neq j_{2}\right)$. Let $\alpha=\operatorname{card}(E)$, since $V$ is infinite-dimensional, $\alpha \geq c_{0}$. Let $X=\left\{\left(e_{1}, e_{2}, \cdots, e_{n} ; \lambda_{1}, \cdots, \lambda_{n}\right): e_{j} \in E, \lambda_{j} \in F, n \in \mathbb{N}\right\}$. For each $n \in \mathbb{N}$ fixed, the number of the selections of choosing $n$ different vectors from $E$ is $\alpha^{n}=\alpha$ (by Theorem 1.7). After choosing $n$ different vectors, each $e_{j}$ multiplies $\lambda_{j} \in F, \beta^{n}=\beta$ possibilities in total, where $\beta=\operatorname{card}(F)$. Thus, the cardinal number of the set consisting of all the linear compositions of $n$ vectors from $E$ is $\alpha \cdot \beta$. It follows that $\operatorname{card}(V) \leq \operatorname{card}(X)=$ $(\alpha \cdot \beta)+(\alpha \cdot \beta)+\cdots+(\alpha \cdot \beta)+\cdots=c_{0}(\alpha \cdot \beta)=\alpha \cdot \beta=\max (\alpha, \beta)$ (applying the result of Remark 2.2, note that $\left.\alpha \geq c_{0}\right)$.

On the other hand, because $V \supset E$ and $V \supset\left\{\lambda e_{1}: \lambda \in F\right\}$, it follows that $\operatorname{card}(V) \geq$ $\operatorname{card}(E)$ and $\operatorname{card}(V) \geq \operatorname{card}(F)$. As a result, $\operatorname{card}(V)=\max (\operatorname{card}(E), \operatorname{card}(F))$.

Proof the Theorem 1.10. " $\Rightarrow$ " suppose $X, Y$ are two infinite sets satisfying $\operatorname{card}(X)=$ $\operatorname{card}(Y)$. Then, there exists a bijection $\phi: X \rightarrow Y . \phi$ induces the map $\Phi: \mathcal{P}(X) \rightarrow \mathcal{P}(Y)$ : $\Phi(E)=\phi(E)=\{\phi(x), x \in E\} \in \mathcal{P}(Y)$, where $E \in \mathcal{P}(x)$. Clearly, $\Phi$ is bijective, so $\operatorname{card}(\mathcal{P}(X))=\operatorname{card}(\mathcal{P}(Y))$. And $\phi$ can also induce the map $\Psi: \mathcal{F}(X \rightarrow \mathbb{Q}) \rightarrow \mathcal{F}(Y \rightarrow \mathbb{Q})$ : $f \rightarrow \Psi(f)=f \circ \phi^{-1}$. It is easy to check that $\Psi$ is an algebra isomorphism from $\mathcal{F}(X \rightarrow \mathbb{Q})$ to $\mathcal{F}(Y \rightarrow \mathbb{Q})$.

$" \Leftarrow$ " In Introduction, we have pointed out that $\mathcal{F}(X \rightarrow \mathbb{Q})$ is an algebra, now denote it by $\mathcal{A}$. Consider a special ideal family of $\mathcal{A}$, denote it by $S$-type: An ideal $\mathcal{J}$ of $\mathcal{A}$ belongs to $S$-type $\Leftrightarrow \mathcal{J}$ satisfies the following conditions:

- (i) $\mathcal{J} \neq \varnothing, \mathcal{J} \neq \mathcal{A}$; 
- (ii) $\mathcal{J}$ is a principal ideal, i.e., $\mathcal{J}=\left\{f_{0} \cdot g: f_{0} \in \mathcal{J}\right.$ is a fixed element, $\left.\forall g \in \mathcal{A}\right\}$;

- (iii) In the family of principal ideals, $\mathcal{J}$ is a maximal element.

It is not hard to prove that all $S$-type ideals are $\left\{\mathcal{J}_{x}: x \in X\right\}$, where

$$
\mathcal{J}_{x}=\{\text { function } f: X \rightarrow \mathbb{Q} ; f(x)=0\} .
$$

Similarly, all S-type ideals of $\mathcal{F}(Y \rightarrow \mathbb{Q})$ are $\left\{\mathcal{J}_{y}: y \in Y\right\}$, where

$$
\mathcal{J}_{y}=\{\text { function } h: Y \rightarrow \mathbb{Q} ; h(y)=0\} .
$$

Since $\Psi: \mathcal{F}(X \rightarrow \mathbb{Q}) \rightarrow \mathcal{F}(Y \rightarrow \mathbb{Q})$ is algebra isomorphism, $\left\{\Psi\left(I_{x}\right): x \in X\right\} \subset\left\{\mathcal{J}_{y}: y \in\right.$ $Y\}$. So $\operatorname{card}(X) \leq \operatorname{card}(Y)$; and because $\left\{\Psi^{-1}\left(\mathcal{J}_{y}\right): y \in Y\right\} \subset\left\{\mathcal{J}_{x}: x \in X\right\}$, it follows that $\operatorname{card}(Y) \leq \operatorname{card}(X)$. In a word, we obtain the result

$$
\operatorname{card}(X)=\operatorname{card}(Y)
$$

by Theorem 1.1.

Remark 2.3. It seems that the requirement in Theorem 1.10 is isomorphism " $\Psi: \mathcal{F}(X \rightarrow$ $\mathbb{Q}) \rightarrow \mathcal{F}(Y \rightarrow \mathbb{Q})$ " is not important. We concern about whether this requirement can be removed. We make following hypothesis.

Hypothesis 2.1. Suppose $X, Y$ are two sets. Then $\operatorname{card}(X)=\operatorname{card}(Y) \Leftrightarrow \operatorname{card}(\mathcal{P}(X))=$ $\operatorname{card}(\mathcal{P}(Y))$.

This hypothesis holds in the following three cases: (1) $X$ and $Y$ are finite sets. (2) the cardinal numbers of $X$ and $Y$ are from a special sequence $\left\{\alpha_{0}, \alpha_{1}, \cdots, \alpha_{n}, \cdots\right\}$, where $\alpha_{0}=\operatorname{card}(S), \alpha_{k}=2^{\alpha_{k-1}}, k \in \mathbb{N}$, and $S$ is an arbitrary infinitely set. (3) If we accept the "continuum hypothesis", then the hypothesis holds when $\max (\operatorname{card}(X), \operatorname{card}(Y)) \leq c$.

\section{Cardinality and separability of the space}

In this section, we discuss the relationship between the cardinality and the separability of the space. A metric space is called separable if it has a countable dense subset.

Theorem 3.1. If $(X, \rho)$ is a separable metric space, then $\operatorname{card}(X) \leq c$.

Proof. Suppose $\left\{x_{n}\right\}_{n=1}^{\infty}$ is a countable dense subset of $X$. Then, for each $x \in X$, there exists a subsequence $\left\{x_{n_{k}}\right\}_{k=1}^{\infty}$ converges to $x$, i.e., $\rho\left(x_{n_{k}}, x\right) \rightarrow 0, k \rightarrow \infty$. Such sequence $\left\{x_{n_{k}}\right\}_{k=1}^{\infty}$ is not unique, but if $x_{1} \neq x_{2}$, it holds that $\left\{x_{n_{k}}^{(1)}\right\} \neq\left\{x_{n_{k}}^{(2)}\right\}$, where $x_{n_{k}}^{(1)} \rightarrow x_{1}$, $x_{n_{k}}^{(2)} \rightarrow x_{2}$. Let

$$
X=\left\{\left\{x_{n_{k}}\right\}_{k=1}^{\infty}:\left\{n_{k}\right\} \text { is a subsequence of } \mathbb{N}\right\}
$$


Each $\left\{x_{n_{k}}\right\}$ corresponds to a real number $t=\sum_{n=1}^{\infty} \frac{\epsilon_{n}}{2^{n}} \in[0,1]$, where

$$
\epsilon_{n}= \begin{cases}1, & n=n_{k} \\ 0, & n \neq n_{k}\end{cases}
$$

Thus, $\operatorname{card}(X) \leq \operatorname{card}(X)=\operatorname{card}([0,1])=c$.

The separability of $(X, \rho)$ does not contribute to the separability of $C(X, \rho)$. For example, Let $X=\mathbb{R}^{n}$ and $\rho$ is the Euclidean metric. Then

$$
\begin{aligned}
& C_{c}\left(\mathbb{R}^{n}\right)=\left\{f \in C\left(\mathbb{R}^{n}\right): f \text { has compact support }\right\}, \\
& C_{0}\left(\mathbb{R}^{n}\right)=\left\{f \in C\left(\mathbb{R}^{n}\right): f \text { vanishes at infinity }\right\} .
\end{aligned}
$$

It is well-known that $C_{c}\left(\mathbb{R}^{n}\right)$ and $C_{0}\left(\mathbb{R}^{n}\right)$ are separable. However, $C\left(\mathbb{R}^{n}\right)$ is not separable. The computation of $\operatorname{card}(C(X, \rho))$ needs further argument.

Theorem 3.2. Suppose $(X, \rho)$ is a separable metric space, $C(X, \rho)$ is the set of all continuous functions with respect to $\rho$. Then, $\operatorname{card}(C(X, \rho))=c$.

Proof. For each $r>0$, construct a function $f_{r} \in C(X, \rho)$ as follow,

$$
f_{r}(x)= \begin{cases}r-\rho\left(x, x_{0}\right), & \text { if } \rho\left(x, x_{0}\right) \leq r \\ 0, & \text { if } \rho\left(x, x_{0}\right)>r\end{cases}
$$

where $x_{0}$ is a fixed point in $X$. So $\operatorname{card}(C(X, \rho)) \geq \operatorname{card}((0,+\infty))=c$. On the other hand, since $(X, \rho)$ is separable, it has a countable dense subset $\left\{x_{n}\right\}_{n=1}^{\infty} \subset X$. Each $f \in C(X, \rho)$, since $f$ is continuous, $f$ is uniquely determined by $\left\{f\left(x_{n}\right)\right\}_{n=1}^{+\infty}$. Let

$$
X=\left\{\left\{\lambda_{n}\right\}_{n=1}^{\infty}: \lambda_{n} \in \mathbb{C}, n \in \mathbb{N}\right\},
$$

then $\operatorname{card}(C(X, \rho)) \leq \operatorname{card}(X)=c^{c_{0}}$.

To complete the proof of Theorem 3.2, we have to prove $c^{c_{0}}=c$. Let

$$
l^{2}=\left\{\left\{\lambda_{n}\right\}_{n=1}^{\infty}: \lambda_{n} \in \mathbb{C}, n \in \mathbb{N} \text { and } \sum_{n=1}^{\infty}\left|\lambda_{n}\right|^{2}<+\infty\right\}
$$

be a separable Hilbert space, by Theorem 3.1, $\operatorname{card}\left(l^{2}\right)=c . l^{2}$ consists of Hilbert cubes $\mathcal{H}=\left\{\left\{\lambda_{n}\right\}_{n=1}^{\infty}: \lambda_{n} \in \mathbb{C}\right.$ and $\left.\left|\lambda_{n}\right| \in I_{n}=\left[-\frac{1}{2^{n}}, \frac{1}{2^{n}}\right], n \in \mathbb{N}\right\}=I_{1} \times I_{2} \times \cdots \times I_{n} \times \cdots$, where $\operatorname{card}\left(I_{n}\right)=c$. It follows that $c=\operatorname{card}\left(l^{2}\right) \geq \operatorname{card}(\mathcal{H})=c^{c_{0}}$. And trivially $c^{c_{0}} \geq c$. Therefore, $c^{c_{0}}=c$.

Remark 3.1. For the equation $c^{c_{0}}=c$, we might have such simpler explanation: since $c=2^{c_{0}}, c^{c_{0}}=\left(2^{c_{0}}\right)^{c_{0}}=2^{c_{0} \times c_{0}}=2^{c_{0}}=c$. However, does $\left(2^{c_{0}}\right)^{c_{0}}=2^{c_{0} \times c_{0}}$ holds? Note that $2^{\alpha}$ is not the usual exponential function! In view of this and Theorem 1.4, Theorem 1.5, we pose the following question: 
Question 3.1. Given arbitrary three cardinal numbers $\alpha_{1}, \alpha_{2}, \beta$ of nonempty sets, whether the equation $\left(\beta^{\alpha_{1}}\right)^{\alpha_{2}}=\beta^{\alpha_{1} \times \alpha_{2}}$ holds?

Consider the space $L^{p}$, which consists of functions defined on a measure space $(X, \mathcal{M}, \mu) . \mu$ is called a complete measure $\Leftrightarrow$ if $E \in \mathcal{M}$ and $\mu(E)=0$, then $\mu(F)=0$ for any subset $F \in \mathcal{M}$ of $E$. There exists a equivalence relation in $\mathcal{M} " \sim ": E_{1} \sim E_{2} \Leftrightarrow$

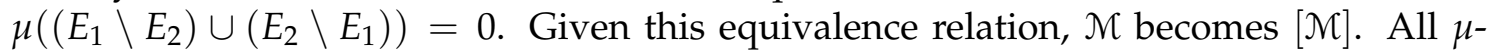
measurable functions on $X$ is denoted by $\mathcal{M}(X)$, define the equivalence relation " $\sim$ ": $f_{1} \sim f_{2} \Leftrightarrow f_{1}-f_{2}=0$, $\mu$-a.e.. The quotient space $\mathcal{M}(X) / \sim$ is denoted by $[\mathcal{M}(X)]$. For $0<p<+\infty$, let

$$
L^{p}(X, \mu)=\left\{f \in[\mathcal{M}(X)]:\|f\|_{p}=\left(\int_{X}|f|^{p} d \mu\right)^{1 / p}<+\infty\right\} .
$$

Define the metric $\rho_{p}$ as follow: for any $f_{1}, f_{2} \in L^{p}$,

$$
\rho_{p}\left(f_{1}, f_{2}\right)= \begin{cases}\left\|f_{1}-f_{2}\right\|_{p}^{p}, & \text { if } 0<p<1 \\ \left\|f_{1}-f_{2}\right\|_{p}, & \text { if } 1 \leq p<+\infty\end{cases}
$$

Then $L^{p}(X, \mu)$ becomes a linear metric space, $0<p<+\infty . L^{\infty}(X, \mu)$ is the set of $\mu$ measurable essentially bounded functions. $L^{\infty}(X, \mu)$ can be a metric space with the metric induced by the essential supremum $\|f\|_{\infty}$.

Theorem 3.3. Suppose $(X, \mathcal{M}, \mu)$ is a measure space with the complete measure $\mu$. Identifying the elements in $\mathcal{M}$ differ by a set of measure zero, we get $[\mathcal{M}]$. If card $([\mathcal{M}]) \geq c_{0}$, then $\operatorname{card}\left(L^{p}(X, \mu)\right) \leq(\operatorname{card}([\mathcal{M}]))^{c_{0}}$, where $0<p<+\infty$.

Proof. Let

$$
\varphi_{\mathbb{Q}}(X, \mu)=\left\{\sum_{j=1}^{n}\left(r_{j}+i r_{j}^{\prime}\right) \chi_{E_{j}}: r_{j}, r_{j}^{\prime} \in \mathbb{Q}, E_{j} \in[\mathcal{M}], n \in \mathbb{N}\right\} .
$$

It is clear that $\varphi_{Q}(X, \mu)$ is dense in $L^{p}(X, \mu), 0<p<+\infty$ (see [1, pp. 200]). Thus, for each $f \in L^{p}(X, \mu)$, there is a sequence of functions $\left\{f_{n}\right\}_{n=1}^{\infty} \subset \varphi_{\mathbb{Q}}(X, \mu)$ satisfying $\left\|f_{n}-f\right\|_{p} \rightarrow 0$, such $\left\{f_{n}\right\}_{n=1}^{\infty}$ is not unique. But if $f \neq g$, it must hold that $\left\{f_{n}\right\} \neq\left\{g_{n}\right\}$, where $\left\|g_{n}-g\right\|_{p} \rightarrow 0$. It follows that $\operatorname{card}\left(L^{p}(X, \mu)\right) \leq\left(\operatorname{card}\left(\varphi_{\mathbf{Q}}(X, \mu)\right)\right)^{c_{0}}$.

And since $\varphi_{\mathbb{Q}}(X, \mu)$ is a linear space over $\mathbb{Q}+i \mathbb{Q},\left\{\chi_{E}: E \in[\mathcal{M}]\right\}=\mathcal{E}$ is its basis, trivially $\operatorname{card}(\mathcal{E})=\operatorname{card}([\mathcal{M}])$, by Theorem 1.7,

$$
\operatorname{card}\left(\varphi_{\mathbf{Q}}(X, \mu)\right)=\max (\operatorname{card}(\mathcal{E}), \operatorname{card}(\mathbb{Q}+i \mathbb{Q}))=\max (\operatorname{card}([\mathcal{M}]), \operatorname{card}(\mathbb{Q}+i \mathbb{Q})) .
$$

And because $\operatorname{card}([\mathcal{M}]) \geq c_{0}=\operatorname{card}(\mathbb{Q})=\operatorname{card}(\mathbb{Q} \times\{0,1\})=\operatorname{card}(\mathbb{Q}+i \mathbb{Q})$, it follows that $\operatorname{card}\left(\varphi_{\mathbb{Q}}(X, \mu)\right)=\operatorname{card}([\mathcal{M}])$. Therefore, we get the result that $\operatorname{card}\left(L^{p}(X, \mu)\right) \leq$ $(\operatorname{card}([\mathcal{M}]))^{c_{0}}$, where $0<p<+\infty$. 
Remark 3.2. For general measure space $(X, \mathcal{M}, \mu)$, whether $\phi_{\mathrm{Q}}(X, \mu)$ is dense in $L^{\infty}(X, \mu)$ is not sure. We must make an assumption of $\mu$. For example, if $\mu(X)<+\infty$, then $\phi_{\mathbb{Q}}(X, \mu)$ is dense in $L^{\infty}(X, \mu)$, so the result of Theorem 3.3 also holds for the case when $p=+\infty$.

Theorem 3.4. Suppose $(X, \rho)$ is a separable metric space, $(X, \mathcal{M}, \mu)$ is a measure space. If $C(X, \rho)$ is dense in $L^{p}(X, \mu)$, then card $\left(L^{p}(X, \mu)\right) \leq c$, where $0<p<+\infty$.

Proof. $(X, \rho)$ is separable, so $\operatorname{card}(C(X, \rho))=c$ by Theorem 3.2. If $C(X, \rho)$ is dense in $L^{p}(X, \mu)$, then using a similar argument to the proof of Theorem 3.3, we can show that

$$
\operatorname{card}\left(L^{p}(X, \mu)\right) \leq(\operatorname{card}(C(X, \rho)))^{c_{0}}=c^{c_{0}}=c .
$$

Now we consider how to make $C(X, \rho)$ dense in $L^{p}(X, \mu)$ ? Use $B\left(x_{0}, r\right)$ to denote the open ball in $X$ of radius $r$ centering at $x_{0} \cdot \mathcal{B}_{X}$ is the Borel algebra generated by open sets in $X$. Suppose $(X, \mathcal{M}, \mu)$ is a measure space, where $\mathcal{M} \supset \mathcal{B}_{X}$ and $\mu$ has the following regular properties: (i) $\exists x_{0} \in X$ s.t. $\mu\left(\bar{B}\left(x_{0}, r\right)\right)<+\infty, \forall r \in[0, \infty)$; (ii) $\forall E \in \mathcal{M}, \mu(E)=$ $\inf \{\mu(V)$ : open set $V \supset E\}=\sup \{\mu(U)$ : closed set $U \subset E\}$.

Theorem 3.5. $(X, \rho)$ is a metric space and $(X, \mathcal{M}, \mu)$ is a measure space. If $\mu$ is regular, then $C(X, \rho)$ is dense in $L^{p}(X, \mu)$, where $0<p<+\infty$.

Proof. In the proof of Theorem 3.3, we have shown that $\phi_{Q}(X, \mu)$ is dense in $L^{p}(X, \mu)$ $(0<p<+\infty)$. Now we consider using continuous functions to approach $\chi_{E}(E \in[\mathcal{M}])$. For any fixed $\epsilon>0$, by the regularity of $\mu$, there exists a closed set $U_{\epsilon}$ and an open $V_{\epsilon}$ such that $U_{\epsilon} \subset E \subset V_{\epsilon}$ and $\mu\left(V_{\epsilon} \backslash U_{\epsilon}\right)<\epsilon$. Let

$$
f_{\epsilon}(x)=\frac{d\left(x, X \backslash V_{\epsilon}\right)}{d\left(x, X \backslash V_{\epsilon}\right)+d\left(x, U_{\epsilon}\right)},
$$

where $d(x, W)=\inf \{\rho(x, y): y \in W\}$ is the distance between $x$ and $W$. By the axiom (iii) of $\rho, d(x, W)$ is continuous function of $x$. It follows that $f_{\epsilon} \in C(X, \rho), 0 \leq f_{\epsilon} \leq 1$ and $\chi_{U_{\epsilon}} \leq f_{\epsilon} \leq \chi_{V_{\epsilon}}$. So

$$
\int_{X}\left|\chi_{E}-f_{\epsilon}\right|^{p} d \mu \leq \mu\left(V_{\epsilon} \backslash U_{\epsilon}\right)<\epsilon, \quad(0<p<\infty) .
$$

Thus, $C(X, \rho)$ is dense in $L^{p}(X, \mu)$, where $0<p<\infty$.

Combining Theorem 3.4 and Theorem 3.5, we get the following corollary.

Corollary 3.1. Suppose $C(X, \rho)$ is a separable metric space, $(X, \mathcal{M}, \mu)$ is a measure space with regular measure $\mu$. Then, $\operatorname{card}\left(L^{p}(X, \mu)\right) \leq c$, where $0<p<+\infty$.

Corollary 3.2. Suppose $\mu$ is a $\sigma$-finite measure of $(X, \mathcal{M}, \mu)$ and $\operatorname{card}([\mathcal{M}])>c$. Then, $L^{p}(X, \mu)$ is not separable, where $0<p \leq+\infty$. 
Proof. First, we assume that $\mu$ is a finite measure, i.e., $\mu(X)<+\infty$. Then, any set $E \in[\mathcal{M}]$ corresponds to a characteristic function $\chi_{E} \in L^{p}(X, \mu)(0<p \leq+\infty)$. Since $\operatorname{card}([\mathcal{M}])>$ $c, \operatorname{card}\left(L^{p}(X, \mu)\right) \geq \operatorname{card}([\mathcal{M}])>c$. Therefore, $L^{p}(X, \mu)$ is not separable. Otherwise, if $L^{p}(X, \mu)$ is separable, by Theorem 3.1, $\operatorname{card}\left(L^{p}(X, \mu)\right) \leq c$. A contradiction!

When $\mu$ is a $\sigma$-finite measure, we can get the result by using the result of the finite measure case, we leave out the details here.

Question 3.2. For general metric space $(X, \mathcal{M}, \mu)$, when $\operatorname{card}([\mathcal{M}])=c$, whether $L^{p}(X, \mu)$ is separable?

We only consider the Euclidean space below. Let $X=\mathbb{R}^{n}, \mu=\mu_{L}$ is the Lebesgue measure. Denote the Lesbesgue measure space by $\mathcal{M}_{L}$. After the process of completion, we get $\left[\mathcal{M}_{L}\right]$. The set of all Lebesgue measurable functions is denoted by $\mathcal{M}_{L}\left(\mathbb{R}^{n}\right)$, given the equivalence relation as before, we get $\left[\mathcal{M}_{L}\left(\mathbb{R}^{n}\right)\right] . C\left(\mathbb{R}^{n}\right)$ represents the set of all continuous functions with respect to Euclidean topology on $\mathbb{R}^{n}$, the dual space of Schwartz space $S\left(\mathbb{R}^{n}\right)$ is the tempered distribution space $S^{\prime}\left(\mathbb{R}^{n}\right)$.

Theorem 3.6. $\operatorname{card}\left(C\left(\mathbb{R}^{n}\right)\right), \operatorname{card}\left(L^{p}\left(\mathbb{R}^{n}\right)\right), \operatorname{card}\left(\left[\mathcal{M}_{L}\left(\mathbb{R}^{n}\right)\right]\right), \operatorname{card}\left(S^{\prime}\left(\mathbb{R}^{n}\right)\right)=c$.

Proof. (i) For $\mathbb{R}^{n}$ is separable, by Theorem 3.2, $\operatorname{card}\left(C\left(\mathbb{R}^{n}\right)\right)=c$.

(ii) For any $r>0$, corresponds to a function $\chi_{B(0, r)} \in L^{p}\left(\mathbb{R}^{n}\right)$, so $\operatorname{card}\left(L^{p}\left(\mathbb{R}^{n}\right)\right) \geq$ $\operatorname{card}((0,+\infty))=c$. On the other hand, since $C\left(R^{n}\right)$ is dense in $L^{p}\left(\mathbb{R}^{n}\right)$ (by Theorem 3.5), where $0<p<+\infty$. It follows that $\operatorname{card}\left(L^{p}\left(\mathbb{R}^{n}\right)\right)=c$ holds when $0<p<+\infty$. The computation of $\operatorname{card}\left(L^{\infty}\left(\mathbb{R}^{n}\right)\right)$ is consisted in the case (iii).

(iii) Let $X_{k}=\left\{f \cdot \chi_{\{k-1 \leq|x| \leq k\}}: f \in L^{\infty}\left(\mathbb{R}^{n}\right)\right\}$. Then $f \in L^{\infty}\left(\mathbb{R}^{n}\right)$ can be written as $f=\sum_{k=1}^{\infty} f_{k}$, where $f_{k} \in X_{k}$ has compact support. Clearly, $X_{k} \subset L^{p}\left(\mathbb{R}^{n}\right)(0<p<$ $\infty)$, so $\operatorname{card}\left(X_{k}\right) \leq \operatorname{card}\left(L^{p}\left(\mathbb{R}^{n}\right)\right)=c$. It follows that $\operatorname{card}\left(L^{\infty}\left(\mathbb{R}^{n}\right)\right) \leq \operatorname{card}\left(X_{1} \times \cdots \times\right.$ $\left.X_{k} \times \cdots\right) \leq c^{c_{0}}=c$; and since $\chi_{B(0, r)} \in L^{\infty}\left(\mathbb{R}^{n}\right), \operatorname{card}\left(L^{\infty}\left(\mathbb{R}^{n}\right)\right) \geq \operatorname{card}((0,+\infty))=c$. Therefore, we get the result that $\operatorname{card}\left(L^{\infty}\left(\mathbb{R}^{n}\right)\right)=c$.

Since $L^{\infty}\left(\mathbb{R}^{n}\right) \subset\left[\mathcal{M}\left(\mathbb{R}^{n}\right)\right], \operatorname{card}\left(\left[\mathcal{M}\left(\mathbb{R}^{n}\right)\right]\right) \geq \operatorname{card}\left(L^{\infty}\left(\mathbb{R}^{n}\right)\right)=c$. On the other hand, each $g \in\left[\mathcal{M}\left(\mathbb{R}^{n}\right)\right]$ can be written as $g=\sum_{k=1}^{\infty} g_{k}$, where $g_{k}=g \chi_{\{x: k-1 \leq|g(x)|<k\}} \in L^{\infty}\left(\mathbb{R}^{n}\right)$. Therefore,

$$
\operatorname{card}\left(\left[\mathcal{M}\left(\mathbb{R}^{n}\right)\right]\right) \leq\left(\operatorname{card}\left(L^{\infty}\left(\mathbb{R}^{n}\right)\right)\right)^{c_{0}}=c^{c_{0}}=c .
$$

(iv) The computation of $S^{\prime}\left(\mathbb{R}^{n}\right)$ is rather complicated, we give an outline of the proof as below: each $u \in S^{\prime}\left(\mathbb{R}^{n}\right)$ corresponds to a sequence of $\left\{u_{k}\right\}$, where $u_{k} \in S^{\prime}\left(\mathbb{R}^{n}\right)_{k}, k \in \mathbb{N}$, where

$$
S^{\prime}\left(\mathbb{R}^{n}\right)_{k}=\left\{u \in S^{\prime}\left(\mathbb{R}^{n}\right): \operatorname{supp}(u) \subset \bar{B}(0, k+1)=\left\{x \in \mathbb{R}^{n}:|x| \leq k+1\right\}\right\} .
$$

It follows that each $S^{\prime}\left(\mathbb{R}^{n}\right)_{k}$ is a subset of tempered distribution with fixed compact support. We define $u_{k}: u_{k}(f)=u\left(\Omega_{k} f\right), \forall f \in S\left(\mathbb{R}^{n}\right)$,

$$
\Omega_{k}(x)=\omega_{k}(|x|)=\omega_{k}\left(\sqrt{x_{1}^{2}+\cdots+x_{n}^{2}}\right)
$$


and $\omega_{k}$ is an one-variable function:

$$
\begin{aligned}
& \omega_{k}(t)= \begin{cases}1, & \text { if } 0 \leq t \leq k, \\
\omega_{0}(t-k), & \text { if } k<t<k+1, \\
0, & \text { if } t \geq k+1,\end{cases} \\
& \omega_{0}(t)=1-\lambda_{0} \int_{0}^{t} \exp (-1 / s(1-s)) d s,
\end{aligned}
$$

where

$$
\lambda_{0}=\left(\int_{0}^{1} \exp (-1 / s(1-s)) d s\right)^{-1} .
$$

Then $\omega_{0} \in C^{\infty}([0,1])$, and

$$
\omega_{0}(0)=1, \quad \omega_{0}(1)=0, \quad \omega^{(k)}(0)=\omega^{(k)}(1)=0, \quad(k \in \mathbb{N}) .
$$

Thus $\Omega_{k}$ has special property: $\left\|\partial^{\alpha} \Omega_{k}\right\|_{\infty} \leq C_{\alpha}$, the constant $C_{\alpha}$ is independent with $k$. We can show that $u_{k}$ weakly converges to $u$, i.e., for any $f \in S\left(\mathbb{R}^{n}\right), \lim _{k \rightarrow \infty} u_{k}(f)=u(f)$. It follows that $\operatorname{card}\left(S^{\prime}\left(\mathbb{R}^{n}\right)\right) \leq \prod_{k=1}^{\infty} \operatorname{card}\left(S^{\prime}\left(\mathbb{R}^{n}\right)_{k}\right)$.

The tempered distribution with compact support $u_{k}$ has Fourier transform $\widehat{u_{k}} \in$ $C^{\infty}\left(\mathbb{R}^{n}\right)$ (see [1, pp. 291-296]). So $\operatorname{card}\left(S^{\prime}\left(\mathbb{R}^{n}\right)_{k}\right) \leq \operatorname{card}\left(C^{\infty}\left(\mathbb{R}^{n}\right)\right)=c$. It follows that $\operatorname{card}\left(S^{\prime}\left(\mathbb{R}^{n}\right)\right) \leq c$. On the other hand,

$$
\operatorname{card}\left(S^{\prime}\left(\mathbb{R}^{n}\right)\right) \geq \operatorname{card}\left(L^{\infty}\left(\mathbb{R}^{n}\right)\right)=c .
$$

Therefore, $\operatorname{card}\left(S^{\prime}\left(\mathbb{R}^{n}\right)\right)=c$.

Remark 3.3. the cardinal number of the Cantor set in $\mathbb{R}$ of zero measure is $c$, and because Lebesgue measure is complete, the cardinal number of the set of all sets of zero measure in $\mathbb{R}$ is $=2^{c}$. Since the union of a non-measurable set and a measurable set is nonmeasurable if they do not intersect, the cardinal number of the set of all non-measurable sets is $=2^{c}$.

Question 3.3. Suppose $\mathcal{M}_{L}$ is the set of all Lesbesgue measurable sets in $\mathbb{R}^{n}$, then all nonmeasurable sets $=\mathcal{P}\left(\mathbb{R}^{n}\right) \backslash \mathcal{M}_{L}$, after adding the equivalence relation (identifying the sets differ by a set of measure zero), denote it by $\left[\mathcal{P}\left(\mathbb{R}^{n}\right) \backslash \mathcal{M}_{L}\right]$, then what is $\operatorname{card}\left(\left[\mathcal{P}\left(\mathbb{R}^{n}\right) \backslash\right.\right.$ $\left.\left.\mathcal{M}_{L}\right]\right)$ ? This is what we will consider next.

\section{Acknowledgements}

Meng Wang is partially supported by NSF of China under Grant No. 11771388. 


\section{References}

[1] G. B. Folland, Real Analysis, Modern Techniques and Their Applications, second Edition, Wiley-Interscience Publication, 2007.

[2] D. X. Xia, Z, R. Wu, S. C. Yan, and W. C. Shu, Real Analysis and Functional Analysis, People's Education Press, 1978.

[3] W. X. Zheng, and S. W. Wang, Real Analysis and Functional Analysis, Nanjing University Press, 1978. 